\title{
ANTROPOLOGÍA
}

\section{LAS CREENCIAS EN TORNO AL NACIMIENTO EN UNA COMUNIDAD ALICANTINA. (UNA COSMOVISIÓN EN TORNO AL NACIMIENTO)}

\author{
$\mathrm{M}^{\mathrm{a}}$ DOLORES LóPEZ GÓMEZ \\ ELENA LÓPEZ TEROL \\ MAR PÉrez LóPEz \\ JOSEFA F. SUCH BOLUDA \\ INMACULADA VIDAGANY ESCRIG
}

\section{RESUMEN}

El sentido del presente estudio ha sido el de intentar captar en cierta medida pequeños rasgos respecto a los conocimientos, valores y creencias que rodean al fenómeno del nacimiento. Concretamente queremos centrarnos en la cosmovisión que planea sobre el recién nacido, enmarcándolo en el ámbito más próximo a nuestra área de actuación como profesionales de la salud.

Las entrevistas se han realizado en una población cercana geográfica y temporalmente a nuestro campo de trabajo, y en ellas hemos descubierto creencias y actitudes que nos han parecido más o menos comprensibles y que representan el modo en que afronta cada cultura las diferentes situaciones de salud.

Pensamos que para poder incidir en nuestra población con programas de educación para la salud del recién nacido en primer lugar hemos de conocer su visión antropológica, pero además no debemos echar por tierra las creencias que resultan beneficiosas sólo por imponer nuestra visión medicalizada del cuidado del recién nacido.

\section{CHILD-BIRTH COSMOVISION}

\section{SUMMARY}

We have tried to capture knowledge, values and beliefs surrounding the phenomenon of child-birth. To be more precise, our aim has been to focus on the cosmovision surrounding the new-born human being, trying to link as much as possible the situation of child-birth with our performance as health professionals.

Interviews have taken place among a population geographically close to our working field. In them, we have discovered beliefs, attitudes, somehow representative of the different ways in which, diverse cultures treat healthcare.

Our feeling is that, if our education programmes for health in new-born babies are to prove effective, then we first have to be acquainted with the population's anthropological vision. 


\section{INTRODUCCIÓN}

El sentido del presente estudio ha sido el de intentar captar en cierta medida pequeños rasgos respecto a los conocimientos, valores y creencias que rodean al fenómeno del nacimiento. Concretamente queremos centrarnos en la cosmovisión que planea sobre el recién nacido, enmarcándolo en el ámbito más próximo a nuestra área de actuación como profesionales de la salud

En ningún caso pretendemos buscar explicaciones a los componentes antropológicos de la cultura que más tarde reseñaremos como hallazgos de nuestro estudio, más bien nuestra tarea será centrarnos en la simple descripción de éstos.

Muchas de estas creencias proceden de una supervivencia milenaria y transcultural de rituales e ideas mágicas y/o religiosas. Se observa que han perdurado a lo largo del tiempo por su valor adaptativo o supervivencial. Intentar racionalizar esta cosmovisión no resultaría sino puro artificio, y, a la vez, tampoco sería conveniente intentar que los usuarios de salud interiorizaran las bases científicas de sus creencias.

\section{MATERIAL Y MÉTODO}

La población objeto de nuestro estudio son las mujeres puérperas y las madres de éstas que encontramos en las plantas de maternidad del Hospital General Universitario de Alicante. Este hospital cuenta con dos plantas dedicadas a puérperas.

A él acuden a dar a luz las mujeres de un área de Alicante y los pueblos de San Vicente, Monforte, Aspe, Guardamar, Torrellano y Agost, además de las mujeres categorizadas de riesgo que vienen remitidas de otros centros hospitalarios de la provincia.

La técnica utilizada en este estudio antropológico ha sido la de la entrevista participante, mediante el uso de un cuestionario semiestructurado con preguntas abiertas y cerradas.

Las entrevistas han sido individuales y realizados por las residentes de matrona. Las entrevistas se han gravado en cinta magnetofónica para que no se escapara ningún detalle de las respuestas, siendo recopiladas y agrupadas posteriormente para su análisis y tratamiento.

Nuestro objeto del estudio es el recién nacido y lo relacionado con la lactancia materna.

Hemos escogido este tema de entre los ofertados para realizar este trabajo antropológico ante todo por los gustos personales y ello basado en que suponíamos que habría menos trabas a la hora de realizar las entrevistas si preguntábamos sobre este tema que sobre otros más íntimos.

El cuestionario está formado por un total de treinta y dos preguntas, primero con siete cuestiones en las que se intentan recoger todos los datos referentes a la filiación y nivel socio-cultural de las mujeres encuestadas. Luego contamos con diez ítems sobre la lactancia materna, seis sobre higiene y cuidados, tres sobre creencias y supersticiones y los seis últimos que versan sobre la elección del nombre, los padrinos y el bautizo.

Casi todas las preguntas han sido extraídas del cuestionario que existe en el anexo del libro "Fenómenos biológicos y fenómenos culturales" perteneciente a la serie de Monografies Sanitaries, Serie D n 10 , Salud para Todos, editado por la Generalitat Valenciana.

A estas se han añadido dos preguntas que creemos interesantes para el estudio:

1. ¿Cree usted que los antojos de la mujer embarazada pueden dar como resultado alguna marca o estigma en la piel?

2. Describir la morfología ¿Tienen relación con el objeto del antojo?

\section{MÉTODO}

Una vez realizadas las encuestas, las respuestas cerradas han sido agrupadas y representadas gráficamente en diagramas de sectores y barras.

Las preguntas abiertas han sido reagrupadas lo máximo posible eso sí, sin obviar palabras y frases testimonio que nos han parecido particularmente significativas.

\section{RESULTADOS}

1.- EDAD DE LA PERSONA ENTREVISTADA:

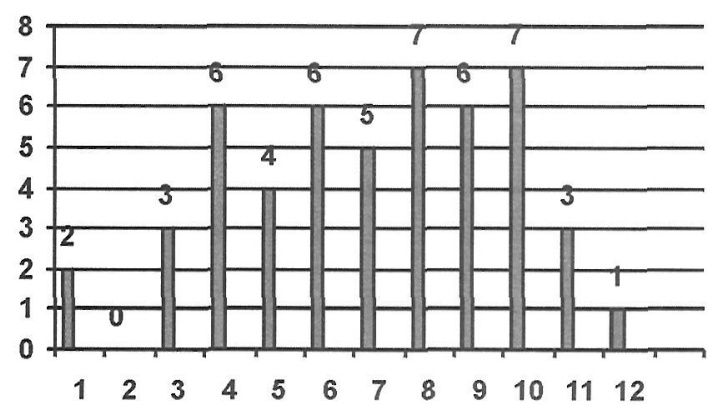




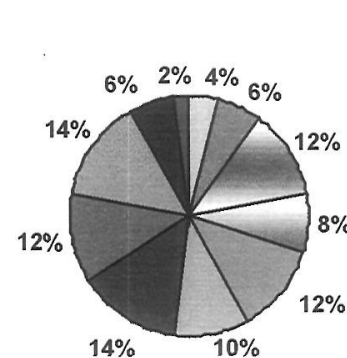

Frecuencia absoluta

1. 25-29 años 2 ....................4\%

2. 30-34 años . 0 .

3. 35-39 años 3

4. 40-44 años 6

5. 45-49 años 6. 50-54 años 7. 55-59 años

8. 60-64 años 9. 65-69 años 10. 70-74 años

11. 75-79 años

12. 80 o más

2.-SEXO: 100\% mujeres.

\section{3.-LUGAR DE NACIMIENTO:}
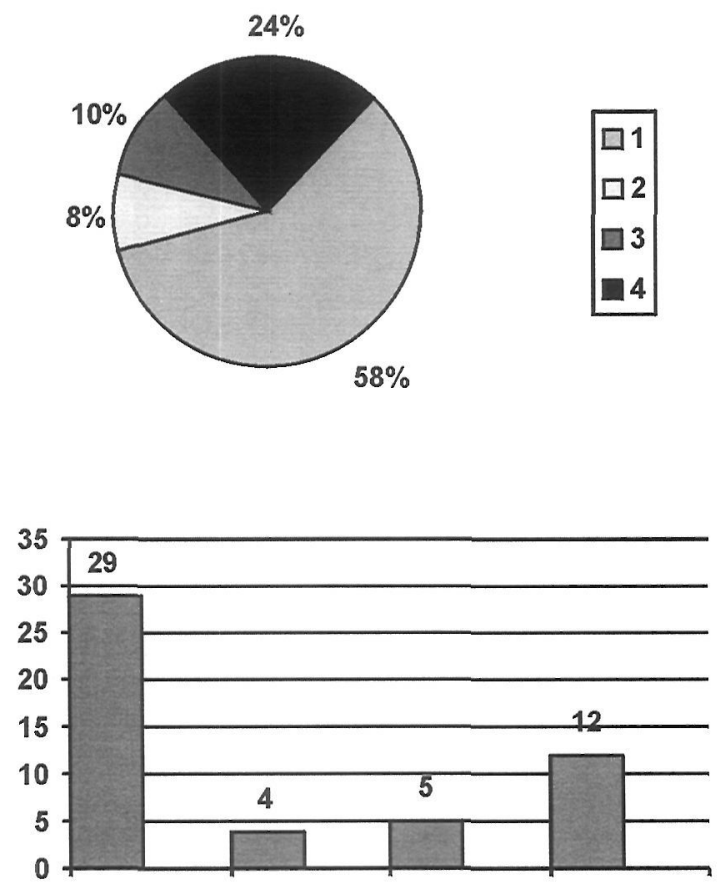

1.-Pueblo en que se

Frec. absoluta

Porcentaje realiza la encuesta 2.-Otra población de la misma comarca. $58 \%$ .29 $.8 \%$ 3.-Otra población del País Valenciano. .4

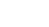
4.-Cualquier otra población. .5 $10 \%$

\section{4.-CUÁNTOS AÑOS LLEVA VIVIENDO EN LA LOCALIDAD:}
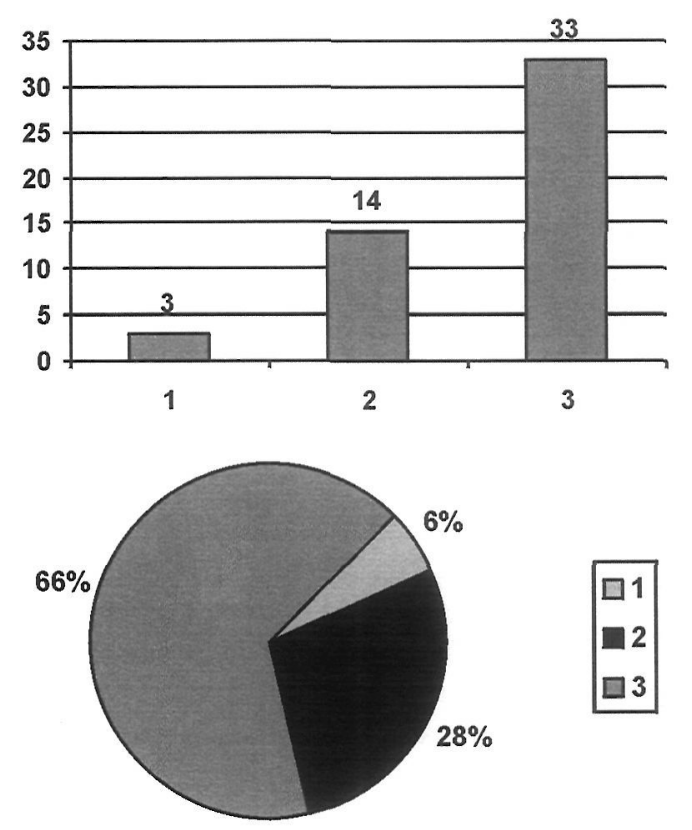

Frec. Absoluta

Porcentaje

1.- Menos de 10 años 3 . $.6 \%$

2.- De 10 a 40 años 14 $.28 \%$

3..- Más de 40 años 33 $.66 \%$

\section{5.-PROFESIÓN DE LA PERSONA ENCUESTADA Totales}

Amas de casa. 42

Estudiantes ..1

Profesional sanitario (aux. Clínica) ..........2

Modista......................................................2

Trabajadora servicios públicos..................1

Empleada de hogar.

6.-ESTÁ: (No se han incluido las amas de casa)

1.- Activo. Frec. Absoluta

2.- Parado 6

3.- Retirado 
7.- NIVEL DE ESTUDIOS DE LA PERSONA ENCUESTADA:
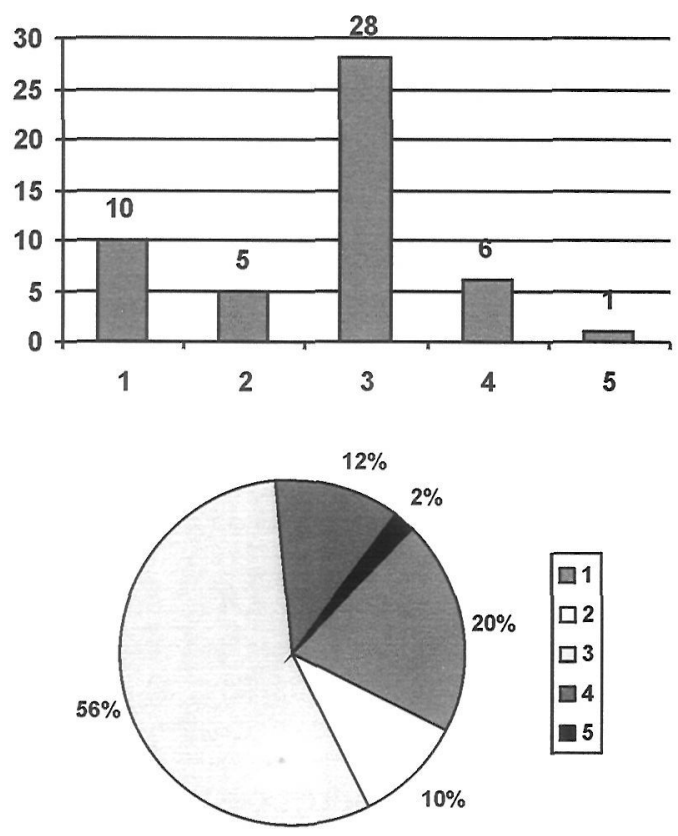

Frec. Porcentaje Absoluta

1.- Analfabetos...............................10...........20\%

2.- No estudios y no clasificable ...5...........10\%

3.- Primarios ............................................56\%

4.- Secundarios .........................................11\%

5.- Superiores................................................

70.- ¿PIENSA QUE LA LACTANCIA MATERNA ES LO MEJOR EN CUANTO A ALIMENTACIÓN PARA EL RECIÉN NACIDO?

Frec absoluta Porcentaje

1.- $\mathrm{Si}$ 50................100\%

2.- No. $0 \ldots \ldots \ldots \ldots \ldots . . . . .0 \%$

3.- NS/NC $0 \ldots \ldots \ldots \ldots \ldots \ldots \ldots$

87.-¿QUUÉ REGLAS SE SIGUEN PARA LA DESIGNACIÓN DE LOS PADRINOS?

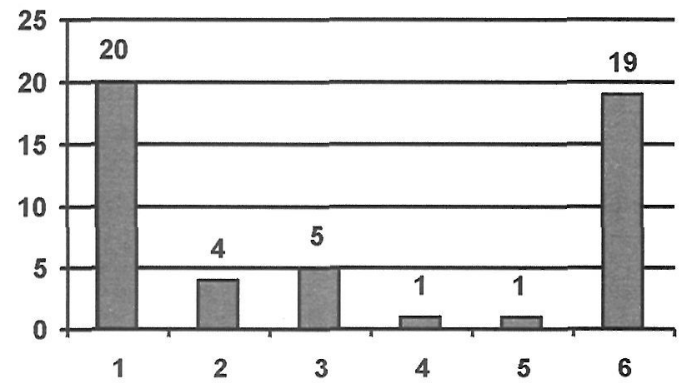

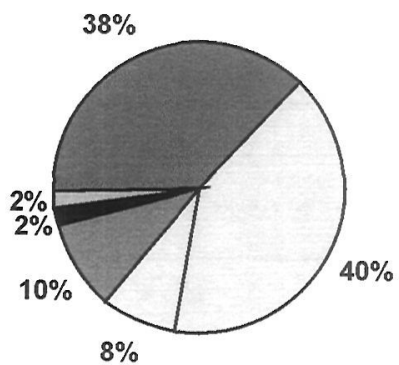

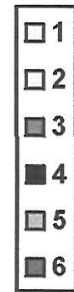

Frec. Porcentaje Absoluta

1.- Los padrinos de boda...........20 $40 \%$

2.- Los abuelos .4 $.8 \%$

3.- Los hermanos solteros de los padres. .5 $.10 \%$ 4.- Los hermanos casados de los padres. $.2 \%$

5.- Personas "acomodadas" $.2 \%$

6.- No hay regla 19 $.19 \%$

88.-¿QUIÉN COMPRA LA ROPA DE CRISTIANAR AL NIÑO?
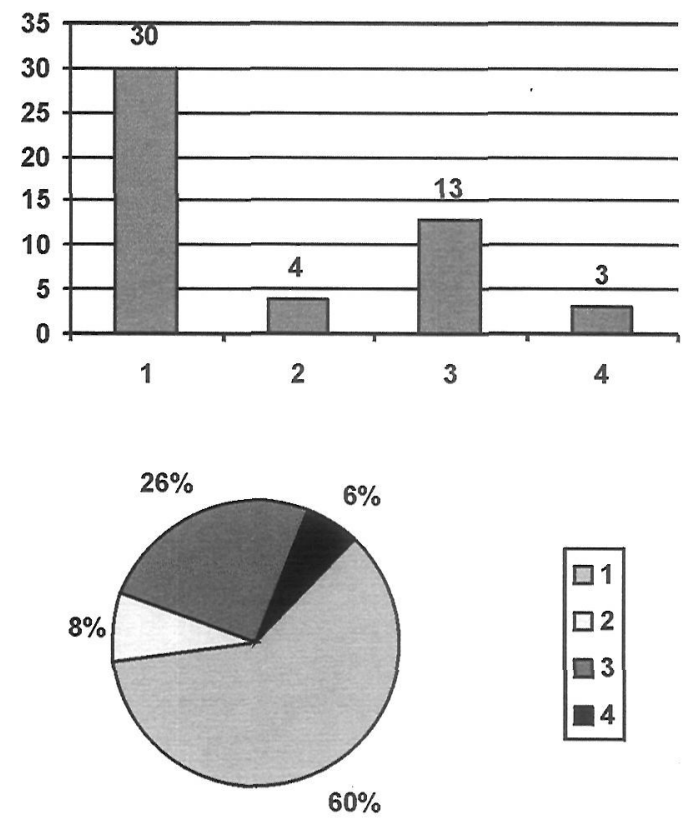

Frec. Absoluta Porcentaje

1.- Los padres 30 . $.60 \%$

2.- Los abuelos 4 $8 \%$

3.- Los padrinos $.26 \%$ 4.- Otros $6 \%$ 
89.-¿CUÁLES SE CONSIDERAN LAS OBLIGACIONES DE LOS PADRINOS?
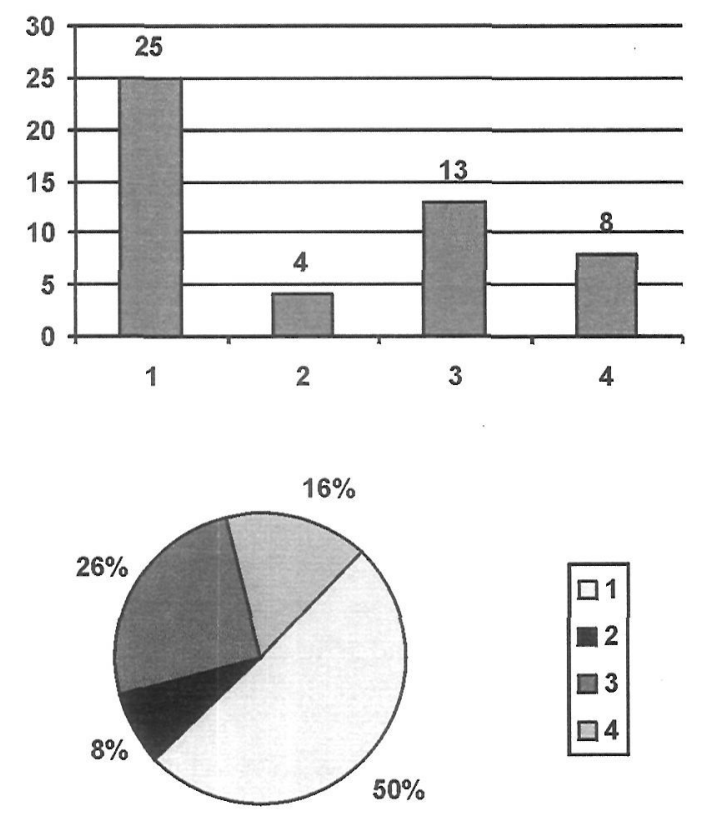

Frec. Porcentaje Absoluta

1.- Cuidar al niño si faltan los padres. .25 $.50 \%$ 2.- Pagar el convite y la ropa de cristianar. .4 $.8 \%$ 3.- Hacer regalos en acontecimientos. 13 $.26 \%$ 4.- No hay ninguna obligación especial.... 8 $.16 \%$

90.-EL NOMBRE DEL NIÑO ¿QUIÉN LO ELIGE?

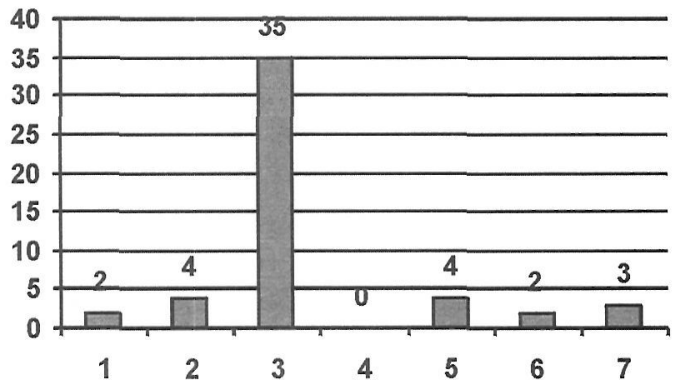

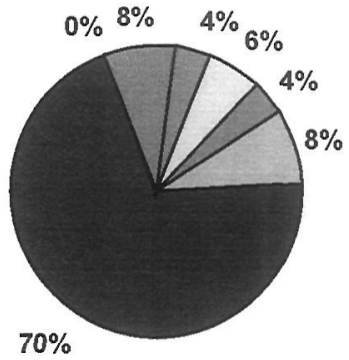

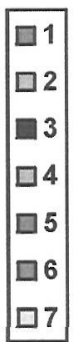

Frec. Porcentaje Absoluta

1.-Padre 2 $.4 \%$

2.-Madre .4 $.8 \%$

3.-Entre los dos 35 $.70 \%$

4.-Abuelos .0 $.0 \%$

5.-Se escoge el santo del día .......4

6.-No hay regla fija. $.8 \%$

7.-Hay una regla fija. .2 $.4 \%$ $.6 \%$

\section{LOS SIGNOS DE GRACIA EN EL RECIÉN NACIDO}

Doce de las mujeres entrevistadas dijeron no conocer ningún signo de gracia en el recién nacido.

Entre las que sí sabían, los más nombrados fueron nacer en Jueves o Viernes Santo y "llorar en el vientre de la madre y si ella no lo cuenta, tendrá gracia". También varias refirieron que nacer con "zurrón", "manto blanco" o "un dedo de grasa".

Otros signos de gracia nombrados fueron:

- Nacer el 16 de marzo (el día de la Cruz)

- Cuando la madre defeca durante el parto justo antes de nacer el niño.

- "Si el niño nace mirando hacia arriba y con las manos abiertas será franco y leal"

- Si es sietemesino

- Nacer con línea alba, cordón de pelo o paño.

- Nacer con una cruz en la barbilla

- "Una verruga o hilito debajo del ojo es signo de buena estrella" (tradición árabe)

- Si tienen un lunar al lado del labio no les falta el amor

- Nacer de pie

- Nacer Jueves Santo y bautizar después del Cirio Pascual.

- Nacer con "telo" en la cabeza

- Nacer con dientes. 


\section{LA LACTANCIA}

Todas las mujeres afirmaron que la lactancia materna es lo mejor para el recién nacido: "lo más natural, cómodo, sano y barato". Casi todas hicieron referencia a que el niño enferma menos porque la madre le pasa defensas y que así se crían mejor, más sanos y gordos. Evita la diarrea, el estreñimiento y le salen dientes más sanos. Algunas también hablaron de que favorece el vínculo madre- hijo y una apuntó que dar el pecho previene el cáncer de mama.

Cuando les preguntamos si conocían algún método para mejorar la cantidad y la calidad de la leche, la mayoría pensaba que en general había que tener una alimentación más sana y aumentar la cantidad de líquidos que se toman (sobre todo leche). Pero en cuanto a alimentos más concretos, los más nombrados fueron el caldo de gallina y de cocido y el caldo de bacalao, también llamado "caldo el cielo" o "bollitori" ( es un hervido con bacalao, tomate, cebolla, judías verdes, patatas y huevo).

Otros alimentos también nombrados fueron los frutos secos, la leche de almendras, el hígado, la carne, la "sustancia de arroz", el pescado hervido, la sopa de pan de ajo y los salazones. También tomar vino y cerveza, aunque en pequeña cantidad.

Se dio también importancia al estilo de vida: tranquilidad, no fumar y no tomar coliflor o alimentos que cambien el sabor de la leche.

Para acortar o alargar la lactancia las respuestas mayoritarias fueron dejar de dar el pecho(acortar) y darle mucho o no dejar de darle para alargarla. Algunas habían oído o sabían por propia experiencia que hay una medicación (pastillas o inyección ) que corta la leche. El estado de ánimo también influye en la duración de la lactancia: el estrés, un susto o un disgusto pueden acabar con ella, y la tranquilidad hace que la leche se mantenga más tiempo.

Una mujer habló de una pomada llamada Asiva, que era amarga y se la ponía en el pezón para que al niño no le gustara la leche.

Seis mujeres explicaron sus rituales para cortar la lactancia:

- Sacarse la leche, ponerla en un paño o en un vaso y tirarla al sol o a la lumbre. Cuando se seca se corta .

- Echar la leche detrás de la puerta y dejar que se seque.
- Ponerse unas llaves huecas en la espalda. Con el frío se corta.

Casi la mitad de las encuestadas no sabían por qué se producía la subida de la leche, aunque dijeron que la madre tenía que beber mucha agua y alimentarse bien para que se produjera. Otras dijeron que porque es "algo natural que sucede después del parto". Unas pocas nombraron las hormonas como causa de la subida.

Siguiendo con el tema de la lactancia, la siguiente pregunta fue si sabían porqué se puede cortar la leche. Las respuestas fueron casi unánimes: por un susto o un disgusto ("igual que se va la regla"). También por tomar medicamentos, si la madre se enferma o si el niño no mama lo suficiente. Concretando las enfermedades maternas una informante dijo "por un aire que le da a la madre, un "pelo" del pecho" y otra "El pelo infecta la leche, las "cañas" y esto se va con compresas calientes").

En cuanto a cuándo hay que empezar a dar de mamar, cada cuánto tiempo y hasta cuando la mayoría argumentaron que enseguida o en las primeras horas de vida, cada 3 horas o cuando el niño lo pide y hasta los 8 o 9 meses o el año.

Hubo otras respuestas que diferían un poco de la opinión generalizada: "Dar de mamar hasta los 4 o 5 meses porque ya empieza a tomar papilla"; "Debe mamar hasta los 5 o 6 años, hasta que tengas leche"; "Se debe esperar unos días antes de ponértelo al pecho, porque los calostros no valen y hasta entonces se le da agua con azúcar".

Casi acabando con el tema de la lactancia preguntamos a las informantes si sabían algún "método casero" para conocer la calidad de la leche. La opinión generalizada, fue que la leche es buena si el niño se queda satisfecho tras la toma y engorda, y es mala si llora entre tomas o pierde peso. También se hizo referencia a la densidad y el color de la leche: la espesa y amarillenta es buena y la cara es mala ("teta gatuna"). Pero se recogieron otras respuestas que merecen ser mencionadas: "Te sacas la leche y la llevas a analizar a la farmacia"; "Si se queda la ropa tiesa y se derrama es buena"; "Una gota de leche en un vaso de agua, si baja en espiral es buena y si se "desparrama" es mala".

Finalmente las entrevistadas pensaban que a través de la leche, la madre le pasa al niño enfermedades (sobre todo catarros y otras enfermedades respiratorias), medicamentos, defensas, tabaco, alco- 
hol y los sentimientos maternos (los disgustos, la tranquilidad). Y se sigue aquí dando importancia a la alimentación materna y sus relación con la producción de leche: si la madre toma alimentos fuertes, los sabores pasan a la leche y de ésta al niño, produciéndole dolor de barriga y "flato" (también si la madre bebe mucha agua )

\section{EL RECIÉN NACIDO}

La primera pregunta sobre este tema fue para conocer los cuidados que nuestras informantes creían que se le debía dar al cordón umbilical: todas destacaron la importancia de mantenerlo limpio y seco, incluso un pequeño número de ellas ( seis) dijeron que no había que bañarlo hasta que se cayera. Los antisépticos más nombrados fueron la mercromina y el alcohol y en menor número el yodo. Bastantes entrevistadas hicieron referencia a los polvos de AZOL, usándolos solos o combinados con un antiséptico para que el cordón se secase antes.

El cordón debía curarse con gasitas y además la mayoría coincidieron en que había que fajarlo con una venda o una fajita ombliguera.

En cuanto al baño y los productos usados para el aseo del recién nacido, la opinión generalizada era que hay que bañarlo todos los días usando un jabón para bebés (o un jabón suave o neutro) y una esponja suave.

Dos o tres informantes dijeron que sólo se le debía lavar cada 2-3 días y sin mojar el cordón ni los huesos de la cabeza.

Una mujer dijo que antes, en el pueblo (Monforte, y también lo comentó otra de Aspe), era la comadrona la encargada de realizar diariamente los cuidados al recién nacido durante el primer mes de vida: lo bañaba, lo vestía y realizaba la cura del cordón umbilical, además de atender las necesidades de la madre.

Se preguntó que porqué creían que los bebés tienen los huesos de la cabeza abiertos: la mayor parte de las encuestadas dijeron que no conocían el motivo. Otras argumentaron que "porque están tiernos" o "no están todavía maduros". Una pequeña parte dijo que "para pasar el parto". En general casi todas las respuestas se relacionaron con la inmadurez del recién nacido: "la mollera es normal"; "les falta calcio"; "Porque están flojos" y "porque hasta que no les da el aire no se les cierra".
Las últimas preguntas en cuanto a cuidados del recién nacido fueron cómo quitar la costra que se les forma en la cabeza y si creían que se les debía abrigar mucho. A la primera pregunta lo más respondido fue que con vaselina ( el aceite de oliva ocupó el segundo lugar en cuanto a preferencias) y despegándola con un peine o peineta cuando se ablandaba. Dos de las informantes respondieron que se quitaba con aceite y aguardiente.

A la segunda pregunta la opinión más común fue que se le debía abrigar según la temperatura que hiciera, igual que a los adultos. Sólo ocho respondieran que era mejor que estuvieran más abrigados que el resto de las personas.

\section{EL MAL DE OJO}

En este tema veintisiete de las encuestadas respondieron que sí que creían, veintidós que no y una no sabía.

Algunas de las que dijeron que sí incluso contaron sus experiencias o las de algún conocido: "A mí se me murió un niño porque una mujer lo besó en la calle. Si le hubiese pegado en aquel momento no habría muerto, porque al llorar no se les revienta la hiel"; "Cuando le echan el mal de ojo el niño se queda pachucho, con la cabeza de lado y se puede morir. Hay que llevarlo a que se lo quiten con el agua y el aceite".

Las personas que echan el mal de ojo lo hacen la mayoría de las veces inconscientemente. El mal lo suelen padecer sobre todos niños "que llaman la atención" (gorditos, guapos,...). La morbilidad asociada suele ser apatía, pérdida del apetito, ictericia ("se ponen amarillos"), tristeza e incluso la muerte del niño si no se cura a tiempo.

Preguntamos sobre las formas de curarlo o de prevenirlo. Para curarlo describieron dos métodos: 1. Hacerle que llore y así se deshace el maleficio. 2. El ritual del agua y del aceite: lo hacen unas mujeres expertas en esto, que no tienen porqué ser curanderas, y que se transmiten este saber unas a otras. Se colocan dos vasos, uno con agua y otro con aceite. Se introduce el dedo del niño mojado en el aceite y luego se deja que el aceite resbale desde el dedo hasta el vaso con agua. Si el aceite queda arriba, flotando, no está aojado y si se mezcla con el agua sí. El ritual se repetía tres días si el niño tenía mal de ojo o si se hacía en viernes, este día valía por dos. 
En cuanto a los métodos para evitarlo, también se describieron varios:

- Colocarle un lazo rojo, visible

- Ponerle una mano de coral rojo.

- Colgarle medallitas.

- Robar pan, ponerlo en un zurrón y que el niño lo lleve colgado.

- Ponerle un escapulario al que la madre le tenga especial fe.

- Poner huesos de cabeza de lagarto en una bolsa..

- Camiseta interior del niño puesta al revés.

\section{LOS ANTOJOS}

Preguntamos si ellas creían en los antojos y en su posible relación con manchas en el recién nacido si no eran satisfechos. Las respuestas afirmativas fueron las mismas que las negativas (23). Las que contestaron que sí, creían además que si no eran satisfechos el bebé nacía con una marca similar a lo que a la madre se le había antojado y además la mancha aparecía donde la madre se tocaba. Una de las informantes nos dijo "se parece a la cosa que te ha llamado la atención, te apetezca o no, porque a lo de dentro sí le apetece".

El alimento más nombrado en cuando a los antojos fueron las fresas.

\section{EL BAUTIZO}

Los padrinos de la boda son les que deben bautizar al primogénito, según veinte de las encuestadas, y 19 de ellas dijeron que no había regla fija. Las obligaciones de éstos son cuidar al niño si faltan los padres (50\% de las encuestadas).

En cuanto a la elección del nombre del recién nacido lo deben elegir entre ambos padres, según la respuesta mayoritaria. Tres informantes respondieron que había una especie de "regla fija" para poner el nombre: "cuando era niña el nombre de la madre o de la abuela y cuando era niño el nombre del padre o del abuelo"; "el padrino elige el nombre del primer hijo y el del segundo lo elige la madre".

Los nombre preferidos suelen ser los de los padres o los familiares y también el del patrono/patrona del pueblo (Remedios, Amparo, José, Juan, Nieves, Inmaculada,...). También el santo del día "si es de mucha devoción". Una de las entrevistadas nos dijo "Eso va por modas, según las telenovelas"

Y para finalizar se pidió que describieran la ceremonia del bautizo: las respuestas obtenidas fue- ron similares independientemente del lugar de origen de la entrevistada: el niño se vestía de blanco, con faldones largos y gorrito. Los padres lo llevaban a la Iglesia, andando, y durante la ceremonia y a la salida de la Iglesia lo llevaba la madrina. Luego se arrojaban monedas (pesetas, céntimos, duros o cinco duros) y peladillas o confituras (peladillas y dinero). Los invitados cantaban "Si no tiren confituras que es muiga la criatura". Luego se solía celebrar un convite o refresco para los familiares e invitados, con bizcochos y dulces.

\section{ANÁLISIS COMPARATIVO}

Hemos analizado la Bibliografía a respecto para poder contrastarla con los resultados obtenidos. Esto queda reflejado en el cuarto apartado de este trabajo.

Después de terminado y analizado nuestro trabajo nos proponemos hacer un análisis comparativo con otros trabajos semejantes en los que se tratan los mismos temas. Así hemos comparado nuestros resultados con los del trabajo de Antonio Limón Delgado realizado en una zona determinada de la comunidad andaluza, y también con los de la Encuesta del Ateneo (1901). Esto nos ha permitido observar que en temas como signos de gracia del recién nacido, el mal de ojo, la lactancia materna y el ceremonial del bautizo las creencias populares suelen coincidir en muchos aspectos, si bien también apreciamos diferencias que confieren a cada estudio un carácter propio por lo que merecen ser tratados por separado cada uno de los aspecto.

\section{Análisis comparativo con el trabajo de Antonio Limón Delgado:}

\section{Signos de gracia:}

En cuanto a los signos de gracia en el recién nacido en el trabajo de Antonio Limón la gente entrevistada diferencia entre los que atribuyen al recién nacido capacidad para curar enfermedades y aquellos que simplemente indican felicidad o prosperidad futura para dicho recién nacido.

En cambio en nuestro trabajo cuando preguntamos sobre este aspecto las informantes atribuyen siempre a la mayoría de los signos capacidad curativa y solo en dos ocasiones se hace mención a signos que son causa de "buena estrella". 
Así pues ambos trabajos coinciden en que los recién nacidos sietemesinos, los que lloran en el vientre materno (y la madre guarda el secreto) y nacer en Jueves Santo tienen la gracia de curar enfermedades y males sobre todo el dolor del bajo vientre denominado en Andalucía "padrejón" y "pará de panxa" en nuestra comunidad.

En cuanto a otros aspectos en torno al nacimiento como nacer de pie, parto laborioso, también se les atribuye el tener gracia futura y no corresponde con el ser rico, feliz o tener larga vida como se les atribuye en Andalucía.

Existen otros signos de gracia que no son mencionados en el estudio de Andalucía y sin embargo se repiten en nuestras entrevistas, como nacer la línea alba, cordón de pelo o nacer con "telo" en la cabeza.

Por otra parte en nuestro trabajo no se hace mención sobre la capacidad de tener gracia en función del número de hijo que se es y sobre el momento y hora del día del nacimiento en contra de las creencias andaluzas donde el quinto hijo de la misma madre tendrá capacidad de curar y domesticar animales.

Por último un aspecto curioso en el que también difieren ambos trabajos es que nuestras entrevistadas no hacen mención a signos de desgracia que sí se dan en Andalucía, tales como nacer en martes o pesar y medir al recién nacido, lo que hace que este sea desgraciado o incluso muera.

\section{Mal de ojo}

En este tema también encontramos coincidencias sobre todo en cuanto a quiénes van a ser propicios para el mal de ojo: niños guapos y gorditos, a través de la mirada y consiste en un cuadro de inapetencia y astenia que puede llevar a la muerte si no se pone solución. Sin embargo lo llamativo en este tema van a ser las diferencias; así en nuestro trabajo el mal de ojo siempre lo va a hacer una mujer y la mayoría de veces de forma inconsciente; en cambio en el trabajo andaluz también serán mujeres pero se menciona sobre todo y con mucha frecuencia a gitanos seguidos de pordioseros, traseúntes e incluso algún vecino enemistado.

En cuanto a la forma de curar el mal de ojo también de detectarlo, en nuestra comunidad es una creencia muy extendida el ritual del agua y aceite que solo es mencionado por una entrevistada en el trabajo de Antonio Limón.
En cuanto a personas capacitadas para curar dicho mal nosotros hemos observado que se habla solamente de persona expertas en dicha función y también de curanderos. En cambio es creencia en Andalucía que la misma persona que los produce lo puede curar, y también van a tener capacidad para ello mujeres que se llamen María u hombres que se llamen Juan, mencionando también al sacerdote como agente sanador.

Por último referido a los amuletos para evitar el mal de ojo se repiten en ambos trabajos muchos de estos como los evangelios o escapularios y trozos de pan seco que se colgaran al recién nacido; sin embargo la colocación de un lazo rojo visible en la ropa del bebé no se menciona en Andalucía y sí se nombra en nuestro trabajo.

\section{Lactancia materna}

Este aspecto no es estudiado por Antonio Limón haciéndose mención al tema de forma muy escueta y en relación a los cuidados de la recién parida.

Se observa similitud en cuanto a cómo debe alimentarse la madre lactante para favorecer la lactancia según nuestro trabajo y según el de Andalucía: en ambos se sombra al caldo de cocido de forma repetida. Observamos también similitud en cuanto a un método para acortar la leche, el cual en nuestro trabajo éste sería colocarse la mujer unas llaves huecas en la espalda mientras que en Andalucía se trata de "unas cuentas de leche que perforadas de un hilo se cuelgan hacia adelante si se quiere leche y hacia atrás para que se retire"

\section{El bautizo: elección de padrinos, nombre del recién nacido, ceremonial.}

En torno al bautizo en lo que respecta a la elección del padrino en Andalucía la respuesta es unánime: serán los padrinos de boda sobre todo en el primogénito siendo su principal obligación costear el convite del bautizo. En nuestro trabajo la mitad de los entrevistados coinciden con la respuesta del trabajo de Andalucía pero difieren en cuanto a la obligación que será cuidar del niño si mueren los padres.

En cuanto a la elección del nombre en ambos trabajos se cita el de los abuelos, el santo del día y en menos ocasiones el de los padres. Es costumbre en Andalucía poner más de un nombre incluso tres nombres siguiendo este orden; no se menciona este hecho en nuestro trabajo. 
Con respecto a la ropa de cristianar coinciden ambos trabajos en que será blanca haciendo especial mención a la prenda del "gorro".

En cuanto al ceremonial resulta curioso observar en los dos trabajos como después de la ceremonia religiosa un cortejo de niños acompañan al recién nacido, padres y padrinos hasta la casa recitando diferentes dichos populares a los que se responde echando monedas por parte de los padrinos.

Un hecho a destacar es la costumbre que se relata en el trabajo de Antonio Limón de que la madrina o el padrino al llegar a casa ofrecen al niño a la madre según diferentes rituales. Sin embargo esta costumbre no se menciona por nuestras entrevistadas.

\section{Análisis comparativo con la encuesta del Ateneo}

\section{Los antojos de la embarazada}

Las respuesta de casi el 50\% de las personas encuestadas y que contestaron que sí creían en los antojos coinciden con los resultados de la encuesta del Ateneo en cuanto a que se trata de satisfacer unos caprichos en sentido de protección hacia el embarazo. Se pretende evitar que los niños salgan marcados en la piel con manchas de diversas formas, tonos y tamaños o malformaciones que afeen su aspecto. Un $73 \%$ de efectos en la criatura se traducen en manchas cutáneas. Un 15\% lo constituyen las deformidades. El antojo más predominante es el deseo de alimentos sólidos comunes y en primer lugar se sitúan las frutas (31\%), carne y pescado o marisco $(8 \%)$, pan $(6 \%)$ y bebidas $(6 \%)$.

Ninguna de nuestras encuestadas hizo la observación de que algún antojo podría ser comer tierra, hierba, yeso, tal y como se hace referencia en la Encuesta del Ateneo (17\%). Tampoco en ninguna encuesta se hace mención del aborto por un antojo insatisfecho en la madre.

\section{Mal de ojo}

Sobre el $52 \%$ de nuestras encuestadas creen en el mal de ojo.

\section{Prevención del mal de ojo:}

De las respuestas obtenidas sólo coincidimos en la encuesta del Ateneo en cuanto a que para prevenir el mal de ojo se siguen utilizando en esta zona medallitas y ponerle al recién nacido escapularios al que la madre le tenga especial fe.

En nuestra zona las madres tienen especial predilección por el color rojo, sobre todo un lazo rojo visible.

La encuesta del Ateneo también menciona al igual que nosotros que el recién nacido que sufre aojamiento se pone enfermo, inapetente, pierde peso y si no se lleva a que le quiten el mal de ojo termina por morir. El método por excelencia para curarlo es el procedimiento de verter algunas gotas de aceite en un vaso de agua. Otra forma de curarlo es hacer que llore el recién nacido y así se deshace el maleficio ( este último método no aparece en la encuesta del Ateneo)

\section{Datos de la Encuesta del Ateneo más relevantes}

- El 99\% son personas las que causan el mal de ojo. El resto pueden ser sapos, lagartijas, comadrejas, etc.

- Suelen ser personas marginadas, brujas, mendigos, gitanas, viejas tuertas o con defectos en la vista, legañosas. Abunda el sexo femenino.

- Pueden contraer el mal los recién nacidos en su inmensa mayoría, el feto (7\%) embarazadas, madres lactantes y mozas de buen ver.

- El aojamiento se origina por la mirada como causa principal seguida de alabanzas verbales y contacto corporal.

- El móvil del que aoja es la envidia sobre todo, la venganza e incluso sin intención.

- Los remedios preventivos de carácter religioso son: colgar de la ropa o del cuello del niño la bolsita de los evangelios, amuleto constituido por un saquito de tela en cuyo interior se coloca un papelito con las primeras palabras de cada uno de los evangelios. En lugar de los evangelios y siguiéndole en frecuencia están la Regla de San Benito, las medallas, las cruces y los escapularios.

- Los remedios preventivos de carácter profano predominan y son I higa de azabache y colgantes de diversos tipos.

- Para detectar y curar el mal de ojo se echan tres gotas de aceite en un vaso de agua: si se divide en muchas gotas, sigue el aojamiento; si las gotas quedan enteras, está hecha la curación. Otros elementos para ver si existe mal de ojo son realizar la medida del aojamiento con un hilo, su pesaje y la comulta de prendas pertenecientes al mismo. 
- Para la curación del mal de ojo predominan ligeramente los consistentes en una lectura de los evangelio y los santiguados, aspersiones con hisopos de agua bendita, las misas, los rezos y ofrendas variadas.

- Las prácticas de tipo profano doblan a las religiosas y entre ellas las que recurren a conjuros, ingestión de líquidos, medida y pesaje del aojado, cortes simbólicos con tijeras, baños rituales, etc.

- La higa de azabache (amuleto especialmente duro) abunda más en Galicia, Asturias, León y Andalucía.

- La bolsita de los evangelios en toda España.

- El diente de ajo (como amuleto que causa se efecto mediante el olor ) en Galicia.

- Los métodos de curación religiosa abundan más en Galicia, Asturias y Vascongadas.

- El trozo de cordón umbilical se conserva como amuleto. En las aldeas vascas se le envuelve en hojas de ajenjo y se le ata al cuello del niño para protegerlo en contra del mal de ojo.

\section{La lactancia}

Alimentación de la puérpera:

Nuestra encuesta coincide con la del Ateneo en los siguientes aspectos:

- Los primeros alimentos que toma la puérpera son los caldos, especialmente de gallina, pollo, cocido o de bacalao.

- En algunas entrevistas apuntaron el tomar vino o cerveza en pequeña cantidad (algo habitual en ciertas zonas de España a principios de siglo)

- El chocolate no lo menciona ninguna de nuestras encuestadas (habitual a principios de siglo)

- Coincidimos con la encuesta del Ateneo en que para estimular la abundancia de leche es necesario que la puérpera tome abundantes líquidos y haga una buena alimentación especialmente caldos, huevos, horchata, etc.

\section{Datos de la encuesta del Ateneo}

- Después de los caldos de gallina los alimentos más frecuentes tomados por la puérpera son dulces, chocolate caliente, bebidas alcohólicas (sobre todo el vino) y puchero de carnero.

- Se evitan los alimentos ácidos y la leche varios días después del parto y sólo se toman caldos y sopas, seguidos de chocolate, vino, carne de pollo y huevos.
- A veces la madre no amamanta a los niños durante dos o tres días y se recurre a otra mujer:"tetera".

- Si a la madre le sale poca leche se le da al niño un pedacito de chocolate envuelto en un trapo para que lo chupe o caldo endulzado con miel o azúcar.

- Para estimular la abundancia de leche se emplean amuletos y talismanes al cuello, siendo los más comunes las cuentas o gotas de leche, abalorios de piedra de color lácteo.

- Entre las clases bajas las madres deben tener cuidado con la pérdida involuntaria de leche. No se deben de dejar alimentos en los platos después de terminar de comer pues éstos se los puede comer otra hembra que esté amamantando perdiendo la primera la leche.

- La madre también puede perder la leche, si por la noche entra una culebra en la casa, le chupa la leche del pecho a la dormida madre y le mete su cola en la boca al infante para que se vaya apaciguando.

- En los Pirineos, si una madre carece inexplicablemente suficiente leche, debe esparcir cenizas alrededor de su cama para observar a la mañana siguiente las huellas delatoras del paso de la serpiente.

- En Andalucía se cree que si le da la luz de la luna a las mantillas o pañales del recién nacido se le seca la leche a la madre.

- El medio más general que la madre emplea para destetar al niño y para cortarse la leche es colocarse en la nuca una llave macho (no hueca) en contraste con una llave hembra (hueca). También se piensa que el líquido se seca al extraerse la leche con las manos y ponerla encima del fuego o de una vela. Ambos métodos aparecen reflejados en nuestras encuestas.

\section{Ceremonial del bautizo}

Según nuestras encuestas (20 de 50) los padrinos de la boda son los que deben bautizar al niño. En la Encuesta del Ateneo se dice que se acostumbra a escoger para padrinos a los parientes, normalmente a los abuelos o parientes cercanos, más que a los amigos. Coincidimos también en el punto de que el la mayoría de lugares se espera que los padrinos, que son los padres espirituales, cuiden del niño en caso de que falten los padres.

No coincidimos con la Encuesta del Ateneo respecto a la elección del nombre: nosotros hemos re- 
cogido que mayoritariamente lo hacen entre los dos padres. En la encuesta se apunta que a menudo el nombre es el de uno de los santos del día en que nació.

\section{Datos relevantes de la encuesta del Ateneo}

- A los niños se le bautiza pronto.

- La elección de padrino se hace cuidadosamente.

- El bautizo se efectúa cuando la madre todavía está en la cama pocos días después del nacimiento. El padre no entra a la Iglesia en aldeas de León y Castilla la Vieja.

- Los padrinos escogidos mantienen el vínculo con su ahijado durante tosa la vida.

- Los métodos para elegir nombre varían mucho según la zona geográfica y con el estatus social de la familia. Si la gente es pobre es común darle un sólo nombre. Cuanto más elevada sea la categoría social de una familia, más nombres tiene la criatura. Uno de los nombres será del santo del día de su nacimiento. La segunda categoría principal serán los nombres de los parientes y de los padrinos.

- Raro es que se les dé a los hijos el nombre de los padres; los de los abuelos se otorgan con frecuencia en Andalucía y en el este de España. A menudo se sigue un orden riguroso en la elección de nombres: el del abuelo o abuela paterno para el primogénito, luego los de los abuelos maternos, después el de los hermanos del padre y por último los de los hermanos de la madre. De ordinario, los padrinos os les dan también sus nombres a los ahijados.

- Cuando finaliza el bautizo sale el grupo de la Iglesia y el padrino tira puñados de monedas y dulces en la puerta donde están aguardando todos los niños del pueblo (no tienen colegio porque el maestro suspende las clases).

- Se espera que los padrinos regalen a la criatura las ropas del bautismo, que paguen en la Iglesia y que ofrezcan un convite para la familia y los amigos que se reúnen después de la ceremonia en la casa de los padres. Durante el convite se ofrecen cigarrillos y puros a los varones y bebidas ligeras y refrescos para todos.

- A veces el padrino le hace regalos a la madre: un pollo, chocolate, pan, dinero, etc.

\section{El recién nacido}

Según la encuesta del Ateneo:

"La comadrona se hace cargo del niño para lavarlo y curarlo y vestirlo, operación que se repite a diario hasta la curación del ombligo".
Curan el cordón envolviéndolo en un trapillo empapado en aceite de oliva hervido o manteca de ganado o de cerdo, espolvoreando la herida con tierra, polvo de tabaco o ceniza de trapo.

$\mathrm{Al}$ infante se le lava y se le viste inmediatamente después del nacimiento, a menudo con los brazos firmemente fajados al cuerpo. Se pone ajo en el agua para bañarlo (en el Noroeste de España).

Al recién nacido se le frota el cuerpo con ajo o se le ata al cuello un diente del mismo para guardarlo de los gusanos intestinales.

Si el niño tarda en respirar debe metérsele en el ano el pico de un pollo, preferiblemente negro. Se supone que el infante absorbe el aliento del ave, que muere asfixiada.

\section{Signos de gracia en el recién nacido}

Según la encuesta del Ateneo:

- Se espera que los niños que nacen con la cabeza cubierta por una membrana tengan riqueza y buena suerte durante toda su vida.

- Es de buena suerte el nacer con los pies delante.

- La gente que nace en años bisiestos presenta una inmunidad natural contra las viruelas y que es de mala suerte nacer en martes.

- En el Este de España se piensa que es muy afortunado quien nace con una membrana en la cabe$\mathrm{za}$, porque a las personas que ven la primera luz de esa manera no pueden penetrarles las balas. De aquí que esas membranas se conservan a menudo, y cuando un joven sale a pelear se lleva un pedacito, como amuleto.

\section{BIBLIOGRAFÍA}

Balaguer, E. (et al) (1996) Fenómenos biológicos y fenómenos culturales. Monografías sanitaries, 10. Valencia.

Limón Delgado, A. Costumbres populares andaluzas de nacimiento, matrimonio y muerte. Diputación Provincial de Sevilla, Sevilla.

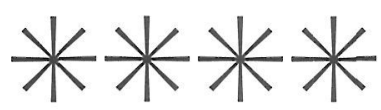

